\title{
Breathing Life into Polycations: Functionalization with pH-Responsive Endosomolytic Peptides and Polyethylene Glycol Enables siRNA Delivery
}

\author{
Martin Meyer,${ }^{\dagger}$ Alexander Philipp,${ }^{\dagger}$ Reza Oskuee,${ }^{\dagger}$ Claudia Schmidt, ${ }^{\ddagger}$ and Ernst Wagner ${ }^{\star}, \dagger$ \\ Pharmaceutical Biotechnology, Department of Pharmacy, Butenandtstrasse 5-13, 81377 München, Department of \\ Physics, Geschwister-Scholl-Platz 1, 80539 München, Center for NanoScience (CeNS), \\ Ludwig-Maximilians-Universität München, Germany
}

Received November 15, 2007; E-mail: ernst.wagner@cup.uni-muenchen.de

siRNA has been widely used to identify gene functions and holds great potential in providing a new class of therapeutics. ${ }^{1}$ The lack of efficient delivery systems is limiting the full therapeutic potential of siRNA technology and represents a challenging hurdle in this field of therapy. For the purpose of nucleic acid transfer, among other synthetic carrier systems, polycations have been applied for the incorporation of plasmid DNA (pDNA) into polyplexes. PolyL-lysine (PLL) was one of the first polycations used for polyplex formation. At physiological $\mathrm{pH}$ the amino groups of PLL are positively charged and interact ionically with the negatively charged nucleic acid which protects the nucleic acid and condenses it into nanoparticles. ${ }^{2}$ An additional asset is the biodegradability of PLL due to its peptidic nature. However, PLL shows almost no siRNA and pDNA transfer activity. Cellular uptake of polyplexes is effective, but the subsequent escape from endosomes into the cytoplasm presents a major bottleneck. Over the past decade polyethylenimines (PEIs) have evolved into currently the most frequently used polycations. In contrast to PLL, PEI can promote its escape to the cytosol from endosomes via the "proton sponge effect", and significant pDNA transfer efficiency can be observed in vitro and in vivo. ${ }^{3,4}$ But in the case of siRNA transfer, PEI like PLL shows only modest delivery activity. Here a modification of these polycations is presented yielding polymers which are effective in siRNA delivery.

The approach includes functionalization with polyethylene glycol (PEG) and a pH-responsive endosomolytic melittin peptide, the major bioactive component of the bee venom. Membrane active peptides like melittin have shown to enhance gene transfer. ${ }^{5,6}$ Lytic activity in the extracellular environment however is unfavorable and triggers toxic side effects. Endosomal acidification is exploited for a triggered activation of the lytic activity in the intracellular compartment. The amines of melittin $(\mathrm{Mel})$ are modified with dimethylmaleic anhydride (DMMAn) which minimizes lytic activity and hence toxicity at extracellular neutral $\mathrm{pH}$. After endosomal acidification the DMMAn protecting groups are cleaved and lytic activity is restored. ${ }^{7,8}$ This principle enables the nucleic acid carrier to adapt its behavior to the different needs of extra- and intracellular delivery steps.

We observed that covalent modification of a polycation with a necessary high density of negatively charged lytic peptides like DMMAn-Mel or influenza peptide can lead to polymer collapse and formation of large aggregates $(>1 \mu \mathrm{m})$ after mixing with nucleic acids. However, small nanoparticles are favorable for delivery. To overcome this drawback, hydrophilic PEG was grafted onto the polycation prior to peptide attachment which allowed formation of small nucleic acid complexes. PEGylation of polyplexes was

\footnotetext{
$\dagger$ Pharmaceutical Biotechnology, LMU München.

¥ Department of Physics, LMU München.
}

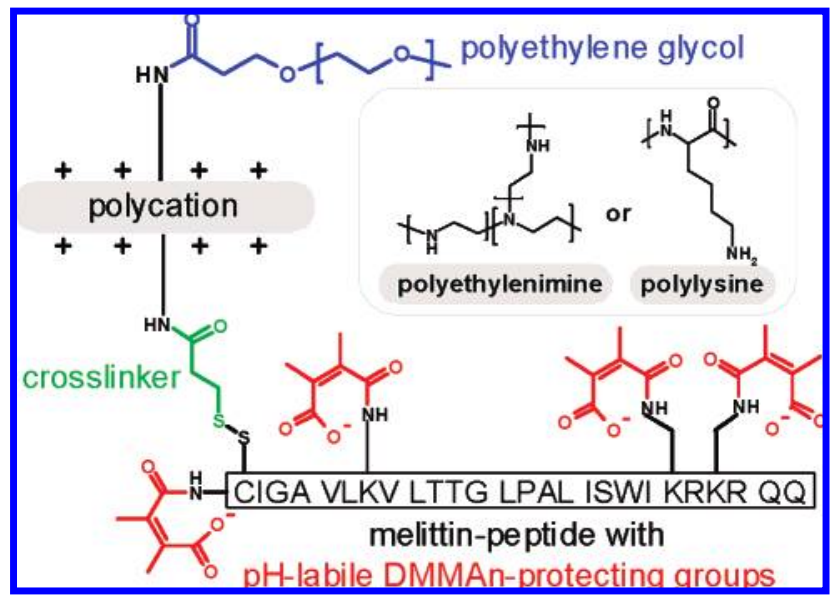

Figure 1. Schematic structure of the $\mathrm{pH}$-responsive endosomolytic polycation-PEG-DMMAn-Mel nucleic acid carrier. Endosomal acidification triggers fast cleavage of the dimethylmaleic anhydride protecting groups and restores lytic activity of melittin.

previously found advantageous in terms of stability and in vivo behavior including pharmacokinetics and tolerability. ${ }^{9}$

PLL (hydrobromide, MW 32 000) and PEI (branched, MW 25 000) were PEGylated with mPEG-succinimidyl propionate (MW 5000). The PEGylated polycations were further modified with heterobifunctional $N$-succinimidyl 3-(2-pyridyldithio)propionate allowing subsequent coupling of DMMAn-Mel peptide via the $\mathrm{N}$-terminal cysteine. The resulting conjugates had molar ratios of approximately 1/1/8 for PLL/PEG/DMMAn-Mel and 1/1,5/8 for PEI/PEG/DMMAn-Mel. A schematic structure of the synthesized carriers is shown in Figure 1.

Interaction of the conjugates with siRNA was confirmed by an ethidium bromide exclusion assay. The small sizes of the siRNA polyplexes are shown in Table 1.

Next, siRNA delivery efficiency of the conjugates was examined with the Neuro 2A-eGFPLuc cell line (mouse neuroblastoma stably expressing luciferase). Luciferase siRNA was complexed with PLL or PEI conjugates in a HEPES-buffered glucose solution.

The experiments were carried out with each polymer bound to 500ng luciferase siRNA at different ratios. $48 \mathrm{~h}$ after initial transfection of 5000 seeded cells, the cells were lysed and assayed for luciferase expression.

As shown in Figure 2, neither PLL nor PEI (with or without PEG) mediated siRNA knockdown. But after modification with DMMAn-Mel siRNA delivery efficiency was greatly enhanced as demonstrated by luciferase gene knockdown. In parallel, complexes with a control siRNA sequence were applied (white bars): if luciferase expression decreases upon transfection with control siRNA (as observed for PLL-PEG), "knockdown" is mainly due to carrier toxicity and can hereby be distinguished from a real 
Table 1. Size of the siRNA Polyplexes ${ }^{a}$

$\begin{array}{lc}\text { PEI/siRNA }(\mathrm{w} / \mathrm{w}=1 / 1, \mathrm{~m} / \mathrm{m}=1 / 1,9) & 26 \pm 8 \mathrm{~nm} \\ \text { PLL } / \mathrm{siRNA}(\mathrm{w} / \mathrm{w}=2 / 1, \mathrm{~m} / \mathrm{m}=1 / 1,2) & 18 \pm 9 \mathrm{~nm} \\ \text { PEI-PEG/siRNA }(\mathrm{w} / \mathrm{w}=1 / 1, \mathrm{~m} / \mathrm{m}=1 / 1,9) & 15 \pm 8 \mathrm{~nm} \\ \text { PLL-PEG/siRNA }(\mathrm{w} / \mathrm{w}=2 / 1, \mathrm{~m} / \mathrm{m}=1 / 1,2) & 7 \pm 1 \mathrm{~nm} \\ \text { PEI-PEG-DMMAn-Mel/siRNA } & 25 \pm 4 \mathrm{~nm} \\ \quad(\mathrm{w} / \mathrm{w}=1 / 1, \mathrm{~m} / \mathrm{m}=1 / 1,9) & \\ \text { PLL-PEG-DMMAn-Mel/siRNA } & 40 \pm 26 \mathrm{~nm} \\ \quad(\mathrm{w} / \mathrm{w}=2 / 1, \mathrm{~m} / \mathrm{m}=1 / 1,2) & \end{array}$

${ }^{a}$ Polycation/siRNA ratio w/w, weight/weight; $\mathrm{m} / \mathrm{m}, \mathrm{mol} / \mathrm{mol}$; size determined by fluorescence correlation spectroscopy.

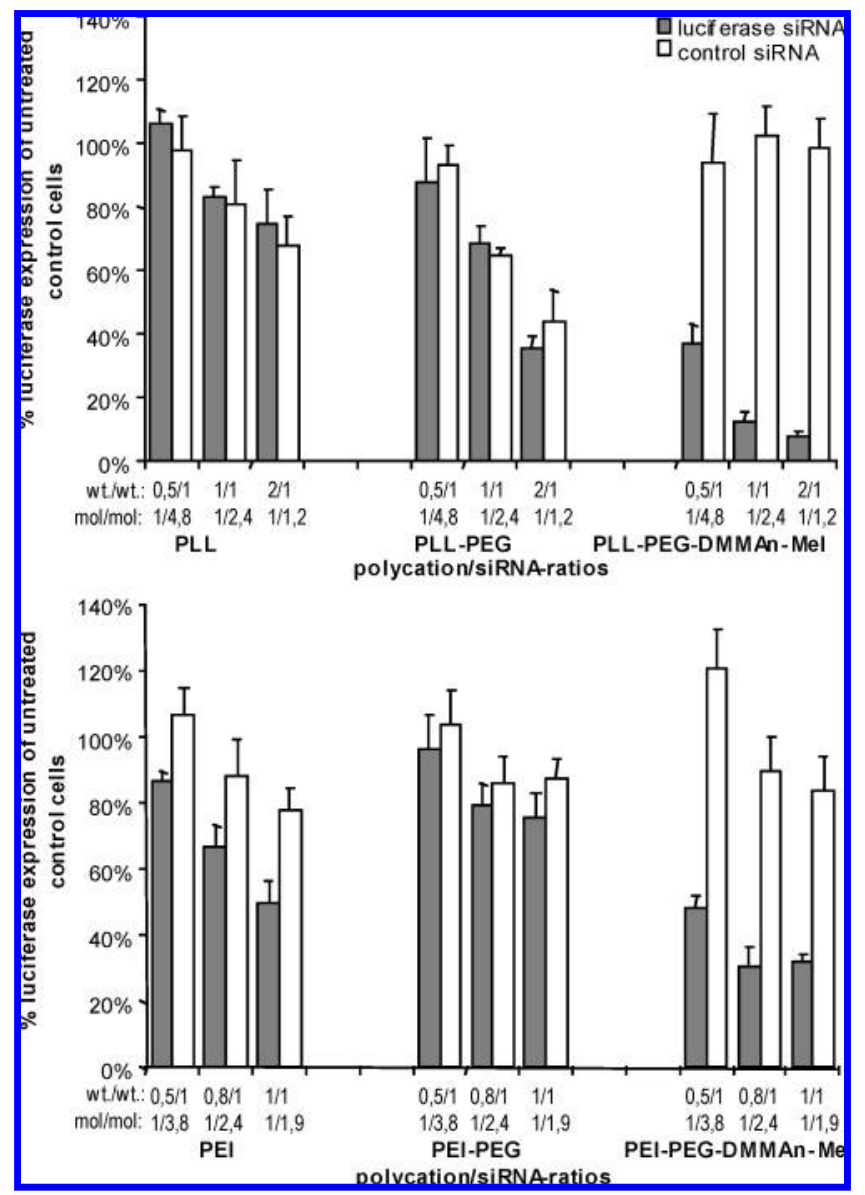

Figure 2. siRNA gene silencing efficiency (gray bars) on stably luciferase expressing Neuro 2A-eGFPluc cells of PLL- and PEI-based conjugates complexed with 500ng siRNA at different polycation/siRNA ratios. White bars indicate transfection with complexes containing control siRNA. Reduced gene expression with control siRNA indicates unspecific knockdown/ carrier toxicity.

siRNA knockdown effect. Metabolic activity was also assayed at the end of transfection and confirmed the high viability of the cells treated with DMMAn-Mel conjugate polyplexes.

The efficiency of pDNA delivery was also tested on Neuro 2A cells (see Supporting Information). Unmodified PEI showed 30fold better gene transfer compared to unmodified PLL. PEGylation had a disastrous effect: PEI lost its gene transfer activity to a background like PEG-PLL. But after attachment of DMMAn-Mel to PEI-PEG gene transfer activity was restored. In the case of PLL modification with PEG and DMMAn-Mel gene transfer was enabled reaching levels similar to those for PEI.

In summary, application of DMMAn-Mel combined with PEG strongly enhanced siRNA delivery efficiency of the polycations. Although the chemistry is different, these findings fit well to recently published work on other bioresponsive and PEGylated siRNA carriers. ${ }^{10-12}$ Remarkably, despite big differences of the unmodified polycations in pDNA transfer activity, pDNA transfer activity of PLL-PEG-DMMAn-Mel was similar to that for the PEI analogue and even better in the case of siRNA transfer (for reasons that remain to be explored). But in contrast to the PEI analogue, PLL has the additional advantage of being biodegradable. As previously shown, conjugation of DMMAn-Mel (in contrast to unmasked melittin, or PEGylation only) considerably lowered the acute cytotoxicity of the polycation PLL. ${ }^{8}$

These findings have implication for the selection of novel polymers for siRNA delivery: polycations with favorable basic characteristics such as nucleic acid binding, compaction, protection, and biocompatibility, but poor delivery activity which obviously would fail in high-throughput screenings ${ }^{13}$ might nevertheless be converted into highly efficient siRNA carriers.

Acknowledgment. This work was funded by the Excellence Cluster "Nanosystems Initiative Munich (NIM)", SFB486 and EC project GIANT.

Supporting Information Available: Experimental details of conjugate synthesis, biological and biophysical characterization; transfection of conjugates with unmodified and succinylated melittin. This material is available free of charge via the Internet at http://pubs.acs.org.

\section{References}

(1) Elbashir, S. M.; Harborth, J.; Lendeckel, W.; Yalcin, A.; Weber, K.; Tuschl, T. Nature 2001, 411, 494-498.

(2) Wagner, E.; Cotten, M.; Foisner, R.; Birnstiel, M. L. Proc. Natl. Acad. Sci. U.S.A. 1991, 88, 4255-4259.

(3) Boussif, O.; Lezoualc'h, F.; Zanta, M. A.; Mergny, M. D.; Scherman, D.; Demeneix, B.; Behr, J. P. Proc. Natl. Acad. Sci. U.S.A 1995, 92, 72977301.

(4) Sonawane, N. D.; Szoka, F. C., Jr.; Verkman, A. S. J. Biol. Chem. 2003, $278,44826-44831$

(5) Ogris, M.; Carlisle, R. C.; Bettinger, T.; Seymour, L. W. J. Biol. Chem. 2001, 276, 47550-47555.

(6) Plank, C.; Oberhauser, B.; Mechtler, K.; Koch, C.; Wagner, E. J. Biol. Chem. 1994, 269, 12918-12924.

(7) Rozema, D. B.; Ekena, K.; Lewis, D. L.; Loomis, A. G.; Wolff, J. A Bioconjug. Chem. 2003, 14, 51-57.

(8) Meyer, M.; Zintchenko, A.; Ogris, M.; Wagner, E. J. Gene Med. 2007, 9, $797-805$

(9) Lee, M.; Kim, S. W. Pharm. Res. 2005, 22, 1-10.

(10) Oishi, M.; Nagasaki, Y.; Itaka, K.; Nishiyama, N.; Kataoka, K. $\underline{\text { J.Am. }}$ Chem. Soc. 2005, 127, 1624-1625.

(11) Rozema, D. B.; Lewis, D. L.; Wakefield, D. H.; Wong, S. C.; Klein, J. J.; Roesch, P. L.; Bertin, S. L.; Reppen, T. W.; Chu, Q.; Blokhin, A. V.; Hagstrom, J. E.; Wolff, J. A. Proc. Natl. Acad. Sci. U.S.A. 2007, 104, $12982-12987$

(12) Murthy, N.; Campbell, J.; Fausto, N.; Hoffman, A. S.; Stayton, P. S Bioconjug. Chem. 2003, 14, 412-419.

(13) Anderson, D. G.; Peng, W.; Akinc, A.; Hossain, N.; Kohn, A.; Padera, R.; Langer, R.; Sawicki, J. A. Proc. Natl. Acad. Sci. U.S.A. 2004, 101, 16028-16033

JA710344V 